\author{
Chen Peijian ${ }^{1}$ \\ State Key Laboratory for Geomechanics \\ and Deep Underground Engineering, \\ School of Mechanics and Civil Engineering, \\ China University of Mining and Technology, \\ Xuzhou 221116, Jiangsu, China \\ e-mail: chenpeijian@cumt.edu.cn; \\ chen_peijian@hotmail.com \\ Chen Shaohua ${ }^{1}$ \\ LNM, \\ Institute of Mechanics, \\ Chinese Academy of Sciences, \\ Beijing 100190, China \\ e-mail: shchen@LNM.imech.ac.cn; \\ chenshaohua72@hotmail.com \\ Yao Yin \\ LNM, \\ Institute of Mechanics, \\ Chinese Academy of Sciences, \\ Beijing 100190, China
}

\section{Nonslipping Contact Between a Mismatch Film and a Finite- Thickness Graded Substrate}

\begin{abstract}
The contact behavior of an elastic film subjected to a mismatch strain on a finitethickness graded substrate is investigated, in which the contact interface is assumed to be nonslipping and the shear modulus of the substrate varies exponentially in the thickness direction. The Fourier transform method is adopted in order to reduce the governing partial differential equations to integral ones. With the help of numerical calculation, the interfacial shear stress, the internal normal stress in the film and the stress intensity factors are predicted for cases with different material parameters and geometric ones, including the modulus ratio of the film to the substrate, the inhomogeneous feature of the graded substrate, as well as the profile of the contacting film. All the physical predictions can be used to estimate the potential failure modes of the film-substrate interface. Furthermore, it is found that the result of a finite-thickness model is significantly different from the prediction of a generally adopted half-plane one. The study should be helpful for the design of film-substrate systems in real applications. [DOI: 10.1115/1.4031936]
\end{abstract}

Keywords: contact mechanics, nonslipping contact, thin film, graded substrate, finitethickness

\section{Introduction}

The mechanical behavior of film/substrate systems is continuously concerned due to the important application in a large number of fields in recent decades [1]. For example, the system of inorganic films on soft substrates is now a hot topic, which can be used to monitor blood pressure during exercise or fabricate electronic eye cameras [2]. A model of film/substrate system can also be adopted in cell mechanics to investigate the interaction between cells and the surrounding substrates [3]. A precise estimation of the interface behavior in a film/substrate system is therefore an essential issue related to many real applications.

Two typically theoretical models have been proposed to study the interface mechanics of film/substrate systems. The first one represented by Akisanya and Fleck [4] and Yu et al [5] can be called as a fracture model, in which a pre-existing crack is introduced at the edge of a thin film. Of course, a model free from cracks or defects would also be crucial in many practical applications [6]. The second one may be addressed as a contact model, in which contact mechanics is used to find the stress field at the interface and near the edge of the film in order to judge possible forming cracks. The governing integrodifferential equations in the film-substrate contact model belong to singular ones, whose exact close-form solutions do not exist [7]. An infinite power series solution was given by Arutiunian [8] for the contact problem between a finite-length stiffener and a half-plane and an alterative method was to express the governing singular equation in terms of the interfacial shear stress [9]. Later, Erodgan and Civelek [10] explored the contact problem between an elastic reinforcement and a homogeneous elastic plate. Multilayered/multiperiodic films bonded to elastic substrates were discussed by Erdogan and Joseph [11,12]. Using the finite difference method, Hu [13] analyzed the film-edge-induced stress in substrates. Shield and Kim [14] discussed the problem of a thin film bonded to an elastic half space by the beam theory considering both normal and shear stresses. A closed form solution to a film bonded a homogeneous

\footnotetext{
${ }^{1}$ Corresponding author.

Contributed by the Applied Mechanics Division of ASME for publication in the Journal of Applied Mechanics. Manuscript received October 7, 2015; final manuscript received November 1, 2015; published online November 13, 2015. Editor: Yonggang Huang.
}

substrate was obtained by Alaca et al. [6] with the assumption of a nearly rectangular film profile and the Vekua's solution procedure of Prandtl's equation.

All the above models involve homogeneous materials. Recently, graded materials have attracted numerous attentions due to the novel and excellent performance [15]. The traditional contact problem between a stamp (punch) and a graded medium has been explored extensively by many researchers to find the contact stress that may cause crack initiation on the contact surface, such as Giannakopoulos and Suresh [16,17], Choi and Paulino [18], Guler and Erdogan [19,20], Ke and Wang [21,22], Mao et al. [23], etc. Considering the interface adhesive effect, the problem of a rigid cylinder/sphere contacting a graded half-space was successfully discussed by Chen et al. [24,25]. The corresponding nonslipping adhesive model and the axis-symmetrically one were solved elegantly by Guo et al. [26,27].

A more complex but practical model is a film bonded on a graded substrate, especially for a deformable film. Guler et al. $[7,28]$ investigated the problem of a film bonded on a graded and graded coated half-space using the approach of a singular integral equation. Whether the result of a film bonded on a graded substrate with an infinite thickness is proper for systems with a finite thickness in practical applications needs further discussion, since a significant thickness effect has been found in the problem of an elastic reinforcement contacting a homogeneous elastic plate by Erodgan and Civelek [10]. Does the thickness of substrates affect the interface behavior of a film-substrate system?

In this paper, a nonslipping contact model is established in order to answer the above questions, in which the mechanical deformation of the film is modeled as a mismatch strain for convenience and the thickness of the graded substrate is finite. The governing equation is formulated analytically, which can be reduced to an integral one and further solved numerically. The interfacial shear stress, the internal normal stress in the film, and the singularity near the contact edges are mainly focused in order to discuss the interface behavior of the film-substrate system.

\section{Contact Model of a Deformable Film and a Finite Thickness Graded Substrate}

The two-dimensional contact model of a deformable film bonded a finite thickness graded substrate with a rigid foundation 
is shown in Fig. 1, where the thickness of the film is $h_{f}$ and the length is $l_{f}=2 a . \mu_{f}$ is the shear modulus of the film and the Poisson's ratio is $\nu_{f}$. Here, the thickness of the film $h_{f}$ is assumed to be a constant, which can also be a function of the $x$ axis [6]. The finite thickness of the graded substrate is $h$ and the shear modulus $\mu_{2}(y)$ is expressed as

$$
\mu_{2}(y)=\mu_{1} \exp (\gamma y)
$$

where the subscript " 1 " denotes the surface layer of the graded substrate, " 2 " the inner of the substrate. $\gamma$ is a constant characterizing the substrate material's inhomogeneity, which can be obtained as

$$
\gamma=\frac{1}{h} \ln \left(\frac{\mu_{3}}{\mu_{1}}\right)
$$

Here, the subscript " 3 " denotes the lower boundary layer of the substrate. The substrate is assumed to have a same constant Poisson's ratio as the film due to a negligible Poisson's ratio effect [16].

\section{Governing Equation of the Boundary Value Problem}

For the present plane contact problem, the constitutive relation of the graded substrate in terms of the displacement components $u(x, y)$ and $v(x, y)$ in $x$ and $y$ directions can be given as

$$
\begin{gathered}
\sigma_{x x}=\frac{\mu_{2}}{\kappa-1}\left[(1+\kappa) \frac{\partial u}{\partial x}+(3-\kappa) \frac{\partial \nu}{\partial y}\right] \\
\sigma_{y y}=\frac{\mu_{2}}{\kappa-1}\left[(1+\kappa) \frac{\partial v}{\partial y}+(3-\kappa) \frac{\partial u}{\partial x}\right] \\
\sigma_{x y}=\mu_{2}\left[\frac{\partial u}{\partial y}+\frac{\partial v}{\partial x}\right]
\end{gathered}
$$

where $\sigma_{i j}(i$ or $j=x$ or $y)$ denotes stress components, $\kappa=3-4 \nu$ for the plane strain case and $\kappa=(3-\nu) /(1+\nu)$ for the plane stress one.

Substituting Eqs. (3a)-(3c) into the classically equilibrium equation yields

$$
\begin{aligned}
&(\kappa+1) \frac{\partial^{2} u}{\partial x^{2}}+(\kappa-1) \frac{\partial^{2} u}{\partial y^{2}}+2 \frac{\partial^{2} v}{\partial x \partial y}+\gamma(\kappa-1) \frac{\partial u}{\partial y} \\
&+\gamma(\kappa-1) \frac{\partial v}{\partial x}=0 \\
&(\kappa-1) \frac{\partial^{2} v}{\partial x^{2}}+(\kappa+1) \frac{\partial^{2} v}{\partial y^{2}}+2 \frac{\partial^{2} u}{\partial x \partial y}+\gamma(3-\kappa) \frac{\partial u}{\partial x} \\
&+\gamma(\kappa+1) \frac{\partial v}{\partial y}=0
\end{aligned}
$$

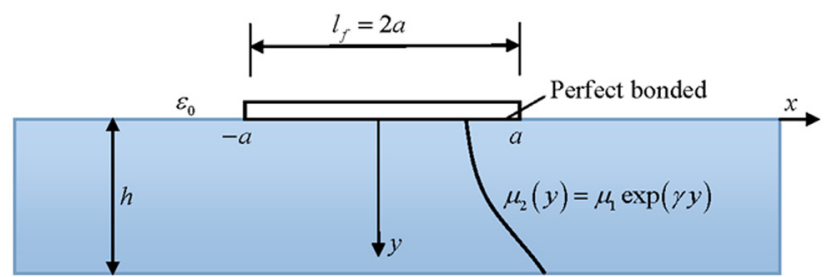

Fig. 1 The perfectly adhesive contact model between an elastic film of length $I_{f}$ and a finite-thickness graded substrate. The variation of environmental temperature induces a mismatched strain at the contact interface. The shear modulus of the substrate abides by a graded law $\mu_{2}(y)=\mu_{1} \exp (\gamma y) ; h$ is the thickness of the graded substrate.
The equation of displacements $u(x, 0)$ and $v(x, 0)$ at the upper surface of the graded substrate can be derived after Fourier transformation to $x$ and a rather lengthy mathematic procedure similar to Chen and Chen $[29,30]$. Here, we skip the details and present only the final formula.

$$
\begin{aligned}
\frac{\partial u(x, 0)}{\partial x}= & \frac{\kappa-1}{4 \mu_{1}} \sigma_{y y}(x, 0)-\frac{\kappa+1}{4 \pi \mu_{1}} \int_{-a}^{a} \frac{\sigma_{x y}(r, 0)}{r-x} \mathrm{~d} r \\
& +\frac{1}{\pi} \int_{-a}^{a}\left[K_{11}(x, r) \sigma_{x y}(r, 0)+K_{12}(x, r) \sigma_{y y}(r, 0)\right] \mathrm{d} r
\end{aligned}
$$

$$
\begin{aligned}
\frac{\partial v(x, 0)}{\partial x}= & -\frac{\kappa-1}{4 \mu_{1}} \sigma_{x y}(x, 0)-\frac{\kappa+1}{4 \pi \mu_{1}} \int_{-a}^{a} \frac{\sigma_{y y}(r, 0)}{r-x} \mathrm{~d} r \\
& +\frac{1}{\pi} \int_{-a}^{a}\left[K_{21}(x, r) \sigma_{x y}(r, 0)+K_{22}(x, r) \sigma_{y y}(r, 0)\right] \mathrm{d} r
\end{aligned}
$$

where the kenerls $K_{i j}(x, r)$ are bounded functions given in the Appendix.

The film thickness $h_{f}$ is assumed to be sufficiently small and the film contacts the graded substrate without any initial stress. Only the shear stress $\sigma_{x y}$ is produced at the contact interface when a mismatch strain is exerted, i.e.

$$
\sigma_{x y}^{f}(x, 0)=\sigma_{x y}(x, 0)= \begin{cases}-f(x), & -a<x<a \\ 0, & x<-a, x>a\end{cases}
$$

The normal stress in the film in the direction of the $y$ axis is

$$
\sigma_{y y}^{f}(x, 0)=\sigma_{y y}(x, 0)=0
$$

Due to a small thickness of the film, the normal stress in the film in the direction of the $x$ axis is assumed to be uniform across the thickness as shown in Fig. 2. The equilibrium equation in the film can be given as

$$
\int_{-a}^{x} \sigma_{x y}^{f}(t, 0) d t+\sigma_{x x}^{f}(x, 0) h_{f}=0
$$

and

$$
\int_{-a}^{a} \sigma_{x y}^{f}(t, 0) d t=-\int_{-a}^{a} f(t) d t=0
$$

Then, the normal stress in the film can be written as

$$
\sigma_{x x}^{f}(x, 0)=\frac{1}{h_{f}} \int_{-a}^{x} f(t) d t
$$

and the strain in the film induced by the interface stress is

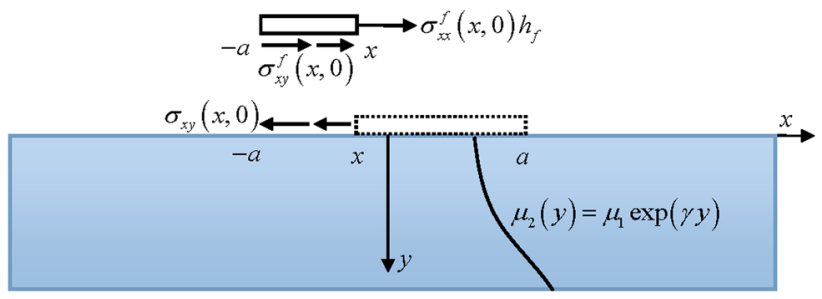

Fig. 2 Schematic of the mechanical behavior of the bonded interface between a deformable film and an elastically graded substrate 


$$
\varepsilon_{x x}^{f}(x, 0)=\frac{\partial u^{f}}{\partial x}=\frac{1+\kappa_{f}}{8 \mu_{f}} \sigma_{x x}^{f}(x, 0)
$$

i.e.

$$
\varepsilon_{x x}^{f}(x, 0)=\frac{1+\kappa_{f}}{8 \mu_{f}} \frac{1}{h_{f}} \int_{-a}^{x} f(t) d t
$$

where $\kappa_{f}=3-4 \nu_{f}$ for the plane strain problem and $\kappa_{f}=$ $\left(3-\nu_{f}\right) /\left(1+\nu_{f}\right)$ for the plane stress one.

Using Eq. (5) leads to the strain at the upper surface of the graded substrate

$$
\varepsilon_{x x}(x, 0)=\frac{\partial u(x, 0)}{\partial x}=\frac{\kappa+1}{4 \pi \mu_{1}} \int_{-a}^{a}\left[\frac{1}{t-x}-\frac{4 \mu_{1}}{\kappa+1} K_{11}(x, t)\right] f(t) \mathrm{d} t
$$

Now consider a film in nonslipping contact with a finite-thickness graded substrate as a reference state. Then, a mismatch strain $\varepsilon_{0}$ is imposed at the interface, which may be induced by a sudden change

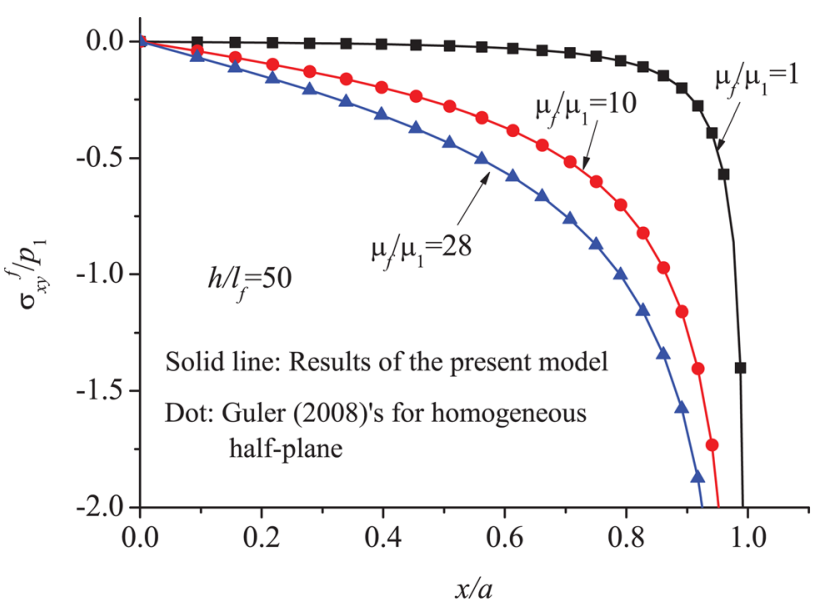

(a)

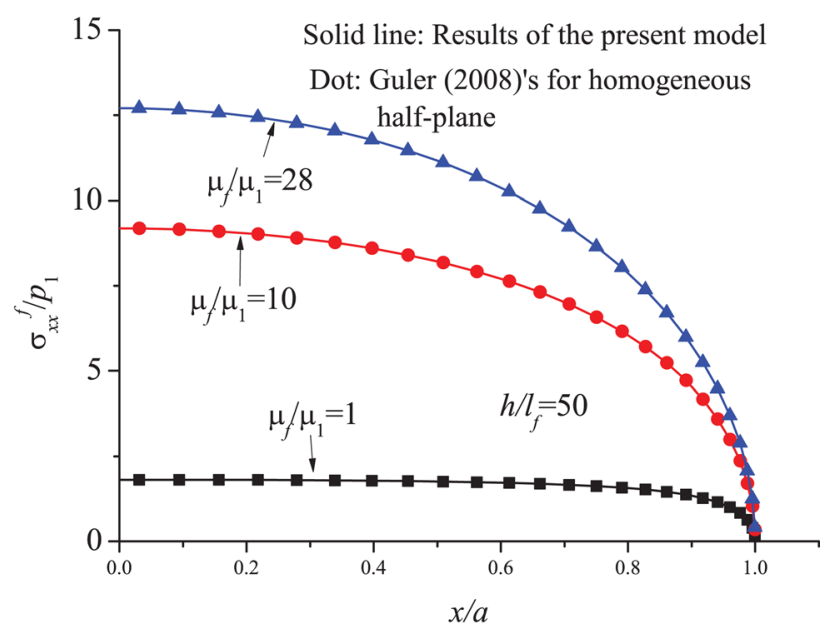

(b)

Fig. 3 Comparison of the distribution of the nondimensional interface shear stress $\sigma_{x y}^{f} / p_{1}$ and the normal stress in the film $\sigma_{x x}^{f} / p_{1}$ for the model of an elastic film in adhesive contact with a homogeneous half-plane and the present one with a relatively thick substrate, where the ratio of the film length to its thickness is $I_{f} / h_{f}=32, \mu_{f}$ is the shear modulus of the film and $\mu_{1}$ denotes the shear modulus of the upper surface of the graded substrate

Journal of Applied Mechanics in temperature in the environment. We have $\varepsilon_{0}=\left(\alpha_{1}-\alpha_{2}\right) \Delta T$, where $\alpha_{i}$ denotes the thermal expansion coefficient of the film or the substrate, $\Delta T$ is the temperature change. The assumption of perfect bonding yields the compatibility condition at the contact interface

$$
\varepsilon_{x x}(x, 0)-\varepsilon_{x x}^{f}(x, 0)=\varepsilon_{0}
$$

Substituting Eqs. (11) and (12) into Eq. (13) yields

$$
\frac{\kappa+1}{4 \pi \mu_{1}} \int_{-a}^{a}\left[\frac{1}{t-x}-\frac{4 \mu_{1}}{\kappa+1} K_{11}(x, t)\right] f(t) \mathrm{d} t-\frac{1+\kappa_{f}}{8 \mu_{f}} \frac{1}{h_{f}} \int_{-a}^{x} f(t) d t=\varepsilon_{0}
$$

which is the governing equation of the present model.

\section{Solution of the Integral Equation}

By introducing the following normalized quantities:

$$
t=a \tau, \quad x=a s
$$

Equations (14) and (8) can be rewritten as

$$
\begin{aligned}
& \frac{1}{\pi} \int_{-1}^{1}\left[\frac{1}{\tau-s}-\frac{4 \mu_{1} a}{\kappa+1} K_{11}(s, \tau)\right] f(\tau) \mathrm{d} \tau-\frac{4 \mu_{1}}{\kappa+1} \frac{1+\kappa_{f}}{8 \mu_{f}} \frac{a}{h_{f}} \int_{-1}^{s} \\
& \quad f(\tau) d \tau=\frac{4 \mu_{1}}{\kappa+1} \varepsilon_{0}
\end{aligned}
$$

Considering the Cauchy-type singular kernel in the integral equation, the solution to Eqs. (16) and (17) may be expressed as [31]

$$
f(s)=\frac{1}{\sqrt{1-s^{2}}} \sum_{n=0}^{\infty} A_{n} T_{n}(s)
$$

where $T_{n}(s)$ denotes the Chebyshev polynomial of the first kind of order $n, A_{n}$ is an unknown constant to be determined.

Substituting Eq. (18) into Eqs. (16) and (17) yields

$$
\begin{gathered}
\frac{1}{\pi} \int_{-1}^{1} \sum_{n=0}^{\infty} A_{n} \frac{T_{n}(\tau)}{\sqrt{1-\tau^{2}}}\left[\frac{1}{\tau-s}-\frac{4 \mu_{1} a}{\kappa+1} K_{11}(s, \tau)\right] d \tau \\
-\frac{4 \mu_{1} a}{\kappa+1} \frac{1+\kappa_{f}}{8 \mu_{f} h_{f}} \int_{-1}^{s} \sum_{n=0}^{\infty} A_{n} \frac{T_{n}(\tau)}{\sqrt{1-\tau^{2}}} d \tau=\frac{4 \mu_{1}}{\kappa+1} \varepsilon_{0} \\
\int_{-1}^{1} \sum_{n=0}^{\infty} A_{n} \frac{T_{n}(s)}{\sqrt{1-s^{2}}} d s=0
\end{gathered}
$$

Considering the following properties of the Chebyshev polynomials:

$$
\begin{aligned}
& \int_{-1}^{1} \frac{T_{n}(s) T_{m}(s)}{\sqrt{1-s^{2}}} d s= \begin{cases}0, & m \neq n \\
1 / 2, & m=n \geq 1 \\
1, & m=n=1\end{cases} \\
& \int_{-1}^{1} \frac{T_{n}(\tau)}{(\tau-s) \sqrt{1-\tau^{2}}} d \tau= \begin{cases}0, & n=0 \\
U_{n-1}(s), & n>0\end{cases}
\end{aligned}
$$


and

$$
\int_{-1}^{s} \frac{T_{n}(\tau)}{(\tau-s) \sqrt{1-\tau^{2}}} d \tau=-\frac{1}{n} U_{n-1}(s) \sqrt{1-s^{2}}
$$

one can readily obtain $A_{0}=0$ and the integral equation may be rewritten as

$$
\sum_{n=1}^{\infty} A_{n}\left[U_{n-1}(s)+Q_{n}(s)\right]=\frac{4 \mu_{1}}{1+\kappa} \varepsilon_{0}
$$

where

$$
\begin{aligned}
Q_{n}(s)= & -\frac{1}{\pi} \int_{-1}^{1} \frac{4 \mu_{1} a}{\kappa+1} K_{11}(s, \tau) \frac{T_{n}(\tau)}{\sqrt{1-\tau^{2}}} d \tau \\
& +\frac{4 \mu_{1} a}{\kappa+1} \frac{1+\kappa_{f}}{8 \mu_{f} h_{f}} \frac{1}{n} U_{n-1}(s) \sqrt{1-s^{2}}
\end{aligned}
$$

Truncating the series in Eq. (18) at $n=N$, and selecting proper collocation points as roots of the following Chebyshev polynomials [32]

$$
T_{N}\left(\tau_{i}\right)=0
$$

the integral equation will be reduced to linear algebraic equations with $N$ unknown constants $A_{n}(n=1,2, \ldots, N)$.

Based on the solution of Eqs. (24) and (25), the shear stress at the contact surface can be approximately given as

$$
\sigma_{x y}^{f}(x, 0)=-\frac{4 \mu_{1} \varepsilon_{0}}{1+\kappa} \sum_{n=1}^{N} A_{n} T_{n}\left(\frac{x}{a}\right) \frac{1}{\sqrt{1-(x / a)^{2}}}
$$
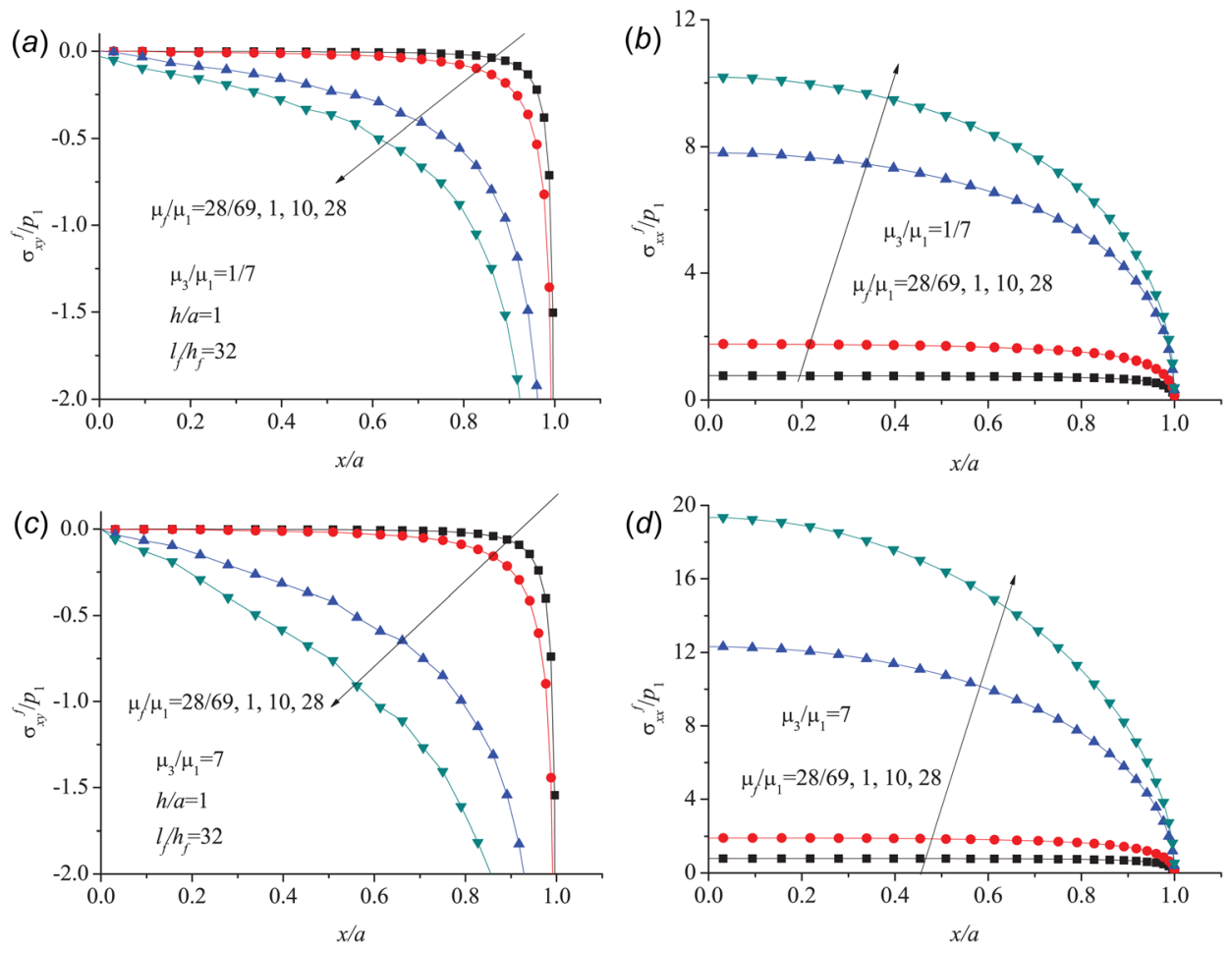

Fig. 4 The distribution of the nondimensional interface shear stress $\sigma_{x y}^{f} / p_{1}$ and the normal stress in the film $\sigma_{x x}^{f} / p_{1}$ for the model of a deformable film in adhesive contact with a finitethickness graded substrate with determined parameters $h / a=1$ and $l_{f} / h_{f}=32$, but with different ratios $\mu_{f} / \mu_{1}$. (a) and $(b)$ for $\mu_{3} / \mu_{1}=1 / 7 ;(c)$ and $(d)$ for $\mu_{3} / \mu_{1}=7$.

021007-4 / Vol. 83, FEBRUARY 2016

Transactions of the ASME 
Table 1 The stress intensity factor $K_{I I} / p_{1} \sqrt{a}$ near the contact edge versus different ratios $\mu_{f} / \mu_{1}$ in cases with fixed parameters $h / a=1, I_{f} / h_{f}=32$, and $\mu_{3} / \mu_{1}$

\begin{tabular}{lcccc}
\hline \hline & $\mu_{f} / \mu_{1}=28 / 69$ & $\mu_{f} / \mu_{1}=1$ & $\mu_{f} / \mu_{1}=10$ & $\mu_{f} / \mu_{1}=28$ \\
\hline $\begin{array}{l}K_{I I} / p_{1} \sqrt{a} \\
\left(\mu_{3} / \mu_{1}=7\right)\end{array}$ & 0.1782 & 0.2793 & 0.7995 & 1.0831 \\
$K_{I I} / p_{1} \sqrt{a}$ & 0.1764 & 0.2722 & 0.6476 & 0.7735 \\
$\left(\mu_{3} / \mu_{1}=1 / 7\right)$ & & & & \\
\hline \hline
\end{tabular}

\section{Results and Discussion}

In the following analysis, the Poisson's ratio of both the film and the substrate is $\nu=0.3$. Only half of the contact area is analyzed due to the symmetric feature of the model.

The film-substrate system with different parameter combinations is investigated. Figures $4(a)$ and $4(b)$ give the distribution of the nondimensional interface shear stress $\sigma_{x y}^{f} / p_{1}$ and the nondimensional normal stress in the film $\sigma_{x x}^{f} / p_{1}$ with a fixed $\mu_{3} / \mu_{1}=1 / 7$ but for different ratios $\mu_{f} / \mu_{1}$, where $p_{1}=\left(4 \mu_{1} \varepsilon_{0} / 1+\kappa\right)$. Figure $4(a)$ shows that the interface shear stress is obviously singular near the contact edges and the singularity increases with an increasing ratio $\mu_{f} / \mu_{1}$, which can be also

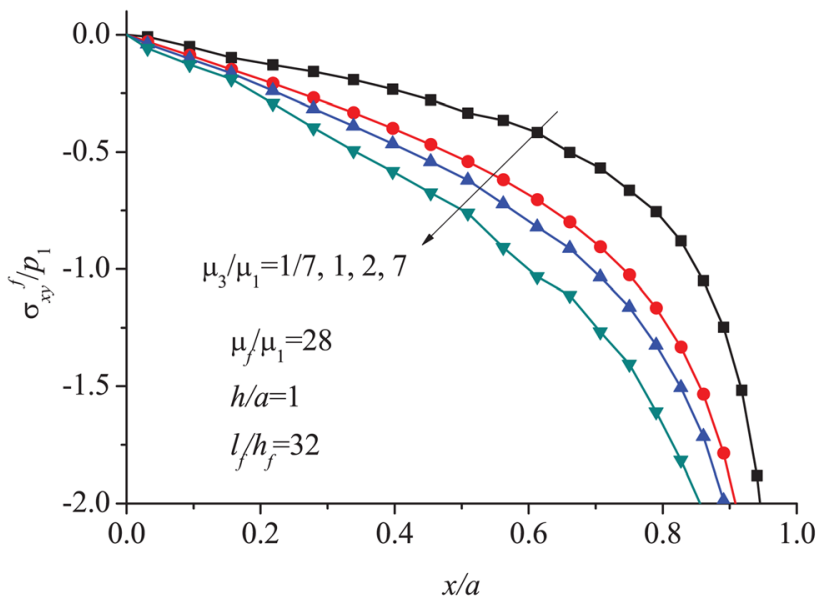

(a)

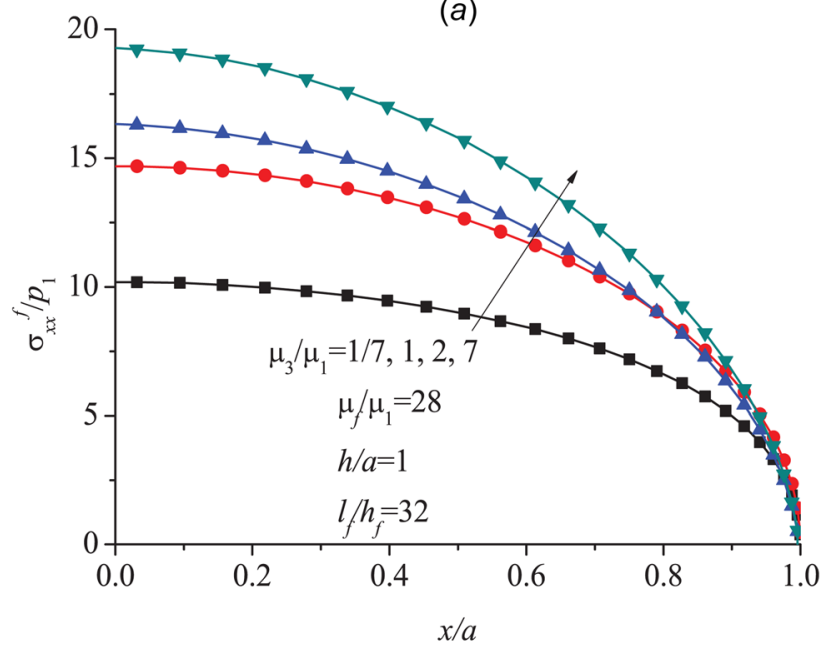

(b)

Fig. 5 The distribution of the interface shear stress $\sigma_{x y}^{f} / p_{1}$ and the normal stress in the film $\sigma_{x x}^{f} / p_{1}$ for the model of a deformable film in adhesive contact with a finite-thickness graded substrate with determined parameters $h / a=1, \mu_{f} / \mu_{1}=28$, $I_{f} / h_{f}=32$, but with different inhomogeneity parameters $\mu_{3} / \mu_{1}$ of the substrate
Table 2 The stress intensity factors $K_{I I} / p_{1} \sqrt{a}$ near the contact edge versus different ratios $\mu_{3} / \mu_{1}$ in cases with determined parameters $\mu_{f} / \mu_{1}=28, h / a=1$, and $l_{f} / h_{f}=32$

\begin{tabular}{ccccc}
\hline \hline & $\mu_{3} / \mu_{1}=1 / 7$ & $\mu_{3} / \mu_{1}=1$ & $\mu_{3} / \mu_{1}=2$ & $\mu_{3} / \mu_{1}=7$ \\
\hline$K_{I I} / p_{1} \sqrt{a}$ & 0.7735 & 0.9409 & 0.9955 & 1.0831 \\
\hline \hline
\end{tabular}

found from the variation of the Mode II stress intensity factor $K_{\mathrm{II}} / p_{1} \sqrt{a}$ shown in Table 1 . The normal stress in the film distributes nonuniformly in the length direction, the maximum value always emerges at the symmetric axis and the normal stress in the film vanishes at the end as shown in Fig. 4(b), which is consistent with the boundary condition. For different ratios $\mu_{f} / \mu_{1}$, the normal stress in the film increases with an increasing value of $\mu_{f} / \mu_{1}$. Both the singularity near the contact edge and the normal stress in the film demonstrate that an interface between a relatively soft film and a determined substrate is much reliable. Figures $4(c)$ and $4(d)$ give the distribution of the nondimensional interface shear stress $\sigma_{x y}^{f} / p_{1}$ and the nondimensional normal stress in the film $\sigma_{x x}^{f} / p_{1}$ for different ratios $\mu_{f} / \mu_{1}$ but with another fixed value $\mu_{3} / \mu_{1}=7$, which means an increasing stiffness along with the depth of the graded substrate. Comparing to Figs. 4(a) and 4(b), it is interesting to find that the varying features of both the shear stress singularity near the contact end and the normal stress in the film do not change.

For a fixed ratio $\mu_{f} / \mu_{1}=28$, the distribution of the interface shear stress $\sigma_{x y}^{f} / p_{1}$ and the normal stress in the film $\sigma_{x x}^{f} / p_{1}$ is shown in Figs. 5(a) and 5(b), respectively, with different values of the inhomogeneity parameter $\mu_{3} / \mu_{1}$. It is found that both the interface shear stress and the normal one in the film increase with an increasing $\mu_{3} / \mu_{1}$. Table 2 gives the Mode II stress intensity factor $K_{\mathrm{II}} / p_{1} \sqrt{a}$, which is obviously enhanced by an increasing $\mu_{3} / \mu_{1}$. That is to say, for a determined film, a substrate with a gradually decreasing stiffness in the depth direction would be advantageous for resisting interface crack initiation. Such a rule should be very useful for the design of new structures and has already been adopted by many nature materials, for example, bone marrow, whose stiffness becomes larger and larger from the inside to the outside.

Figure 6 gives the effect of thickness of the graded substrate on the distribution of the interface shear stress and the normal stress in the film with a fixed ratio $\mu_{f} / \mu_{1}=28$. Figures $6(a)$ and $6(b)$ correspond to cases of a finite-thickness substrate with an increasing stiffness in the depth direction $\mu_{3} / \mu_{1}=7$, while Figs. $6(c)$ and $6(d)$ correspond to the one with the stiffness decreasing in the depth direction $\mu_{3} / \mu_{1}=1 / 7$. The substrate thickness shows a more regular effect on the interface shear stress and the normal stress in the film in the former case, where both stresses increase monotonically with a decreasing thickness of the graded substrate. However, nonmonotonically variation in the interface shear stress and the normal stress in the film can be found in Figs. 6(c) and $6(d)$. Variation of the Mode II stress intensity factor $K_{\text {II }} / p_{1} \sqrt{a}$ versus the nondimensional substrate thickness $h / a$ is given in Fig. 7 for different $\mu_{3} / \mu_{1}$, where the result of a homogeneous halfplane case is also shown for comparison. Obviously, $K_{\mathrm{II}} / p_{1} \sqrt{a}$ increases monotonically with a decreasing thickness $h / a$ in cases of $\mu_{3} / \mu_{1} \geq 1$, while decreases first and then increases for the case of $\mu_{3} / \mu_{1}=1 / 7$. All the results demonstrate that the effect of substrate thickness on the interface behavior can not be neglected. A semi-infinite model may not be precise in explaining phenomena found in a finite film-substrate structure. However, if the substrate thickness is large enough, the boundary effect should be weakened in all cases as shown in Fig. 7, both a half-plane model and a finite one may replace each other.

The ratio effect of the film length to its thickness in the finite model on the distribution of the nondimensional interface shear stress $\sigma_{x y}^{f} / p_{1}$ and the normal stress in the film $\sigma_{x x}^{f} / p_{1}$ is shown in Fig. 8. A fixed value $\mu_{3} / \mu_{1}=1 / 7$ is adopted in Figs. 8(a) and 

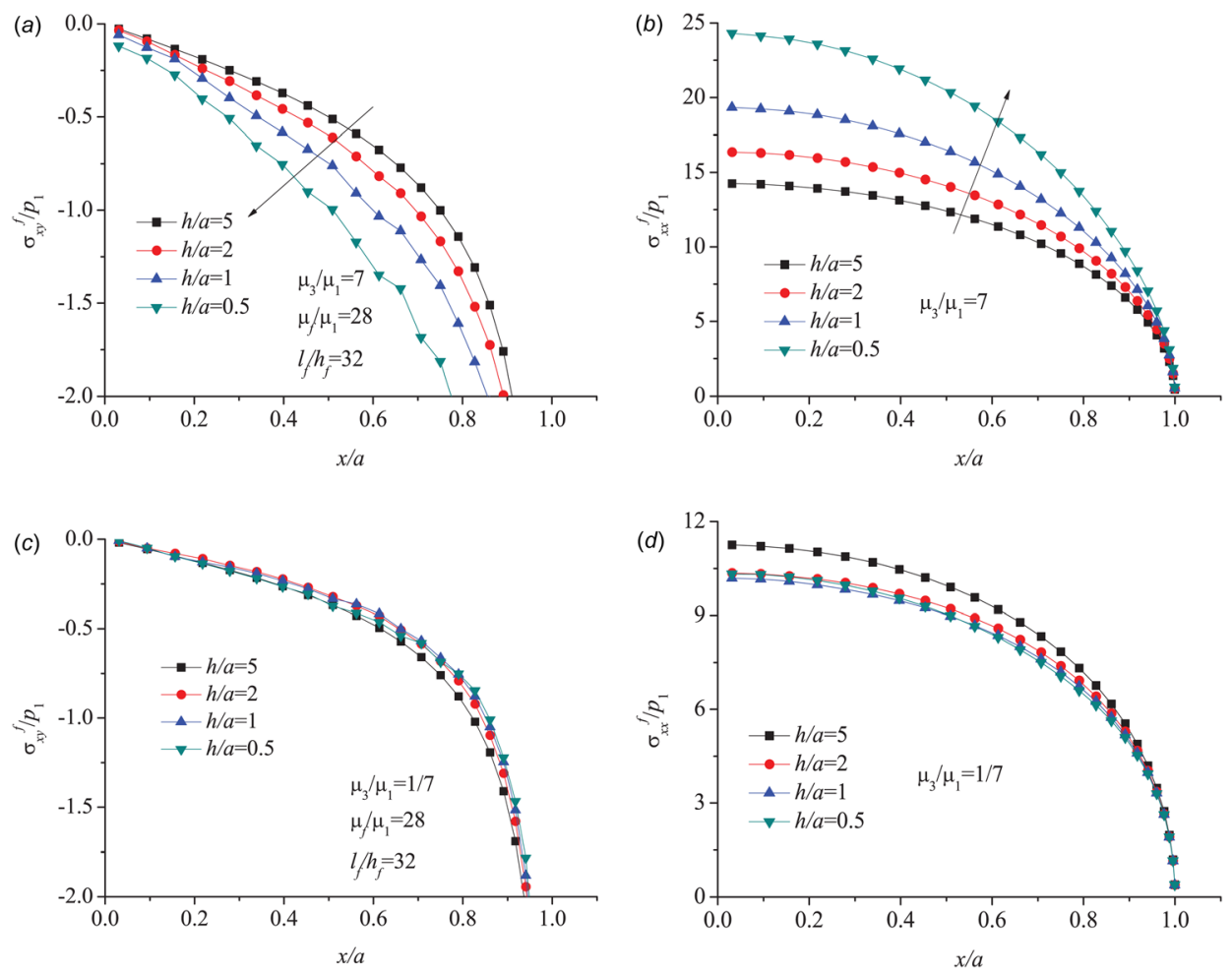

Fig. 6 The distribution of the interface shear stress $\sigma_{x y}^{f} / p_{1}$ and the normal stress in the film $\sigma_{x x}^{f} / p_{1}$ for the model of a deformable film in adhesive contact with a finite-thickness graded substrate with determined parameters $\mu_{f} / \mu_{1}=28, I_{f} / h_{f}=32$, but with different ratios $h / a$. (a) and $(b)$ for $\mu_{3} / \mu_{1}=7 ;(c)$ and (d) for $\mu_{3} / \mu_{1}=1 / 7$.

$8(b)$, while $\mu_{3} / \mu_{1}=7$ is used in Figs. 8(c) and $8(d)$. Both cases show that the interface shear stress decreases with an increasing ratio of $l_{f} / h_{f}$. The corresponding Mode II stress intensity factor $K_{\mathrm{II}} / p_{1} \sqrt{a}$ is given in Table 3 , which also exhibits a decreasing singularity with an increasing ratio $l_{f} / h_{f}$. It means that, in cases with a fixed film length, the thinner the film, the stronger the interface strength is; while for a fixed film thickness, the shorter the film, the more possible failure at the contact end it is. However, in cases with a fixed film thickness, Figs. $8(b)$ and $8(d)$ show that the normal stress in the film increases with an increasing film length no matter $\mu_{3} / \mu_{1}$ is larger or smaller than 1 . Therefore, a longer film is more likely to break at site of the symmetric axis than a shorter one in cases of films with a fixed thickness.

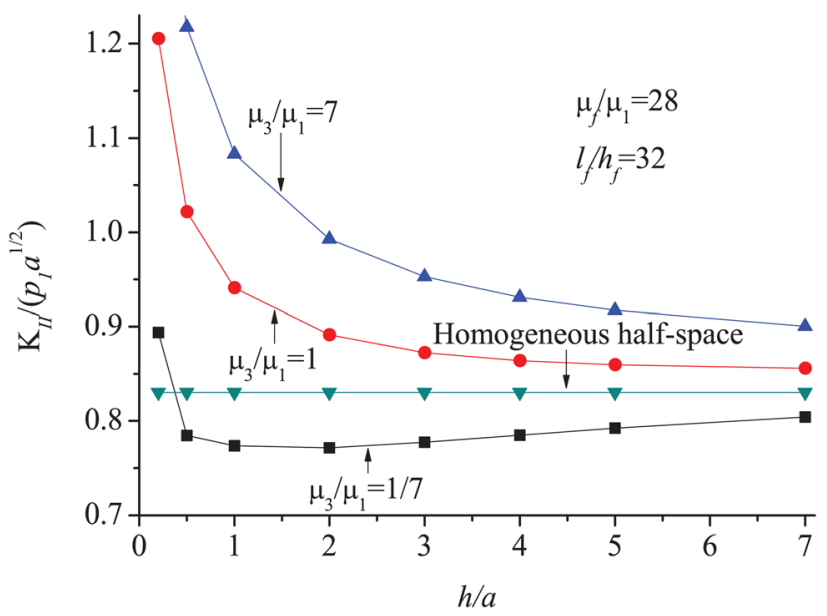

Fig. 7 Variation of the Mode II stress intensity factor $K_{I I} / p_{1} \sqrt{a}$ versus the ratio of the substrate thickness to half of the film length $h / a$ for different ratios $\mu_{3} / \mu_{1}$, where $\mu_{f} / \mu_{1}=28$
All the above analysis is based on an assumption of a uniform film thickness. If the profile of the film in the thickness direction is a function of the $x$ axis, the present model may be transformed to be the model by Alaca et al. [6]. In Alaca et al. [6], the profile function of the film in the thickness direction was adopted as

$$
h_{f}(x)=h_{f 0} \sqrt{1-\frac{x^{2}}{a^{2}}}\left(1+\lambda \frac{x^{2}}{a^{2}}\right)
$$

where $h_{f 0}$ denotes the film thickness at $x=0 . \lambda$ is a shape parameter. Tuning the parameter $\lambda$ would lead to different film profiles as shown in Fig. 9, which may change from a convex shape to a concave one with an increasing $\lambda$. With $\lambda=0.9, \gamma=0$ and $h \gg a$, the present model can be reduced to the one by Alaca et al. [6], where the stress intensity factor was defined as

$$
K_{2}=\lim _{x \rightarrow-a} \sigma_{x y}^{f}(x, 0) \sqrt{2 \pi(x+a)}
$$

Substituting $\mu_{f} / \mu_{1}=28, l_{f} / h_{f 0}=32$ into Eq. (32) and using the thickness profile in Eq. (31) yield $K_{2}=0.4683$, which is consistent well with the result of Alaca et al. The requirement that the thickness of the film is much less than its length should be satisfied since the normal stress in the film is assumed to distribute uniformly in the thickness direction.

\section{Summary}

A nonslipping contact model between a mismatched film and a finite-thickness graded substrate is investigated in the present paper. Fourier transform method is used to transform partial differential equations to the integral ones. Distributions of the interface shear stress, the normal stress in the film, and the stress intensity factors near the contact edge are analyzed. It is found that the interface behavior would be influenced significantly by material 

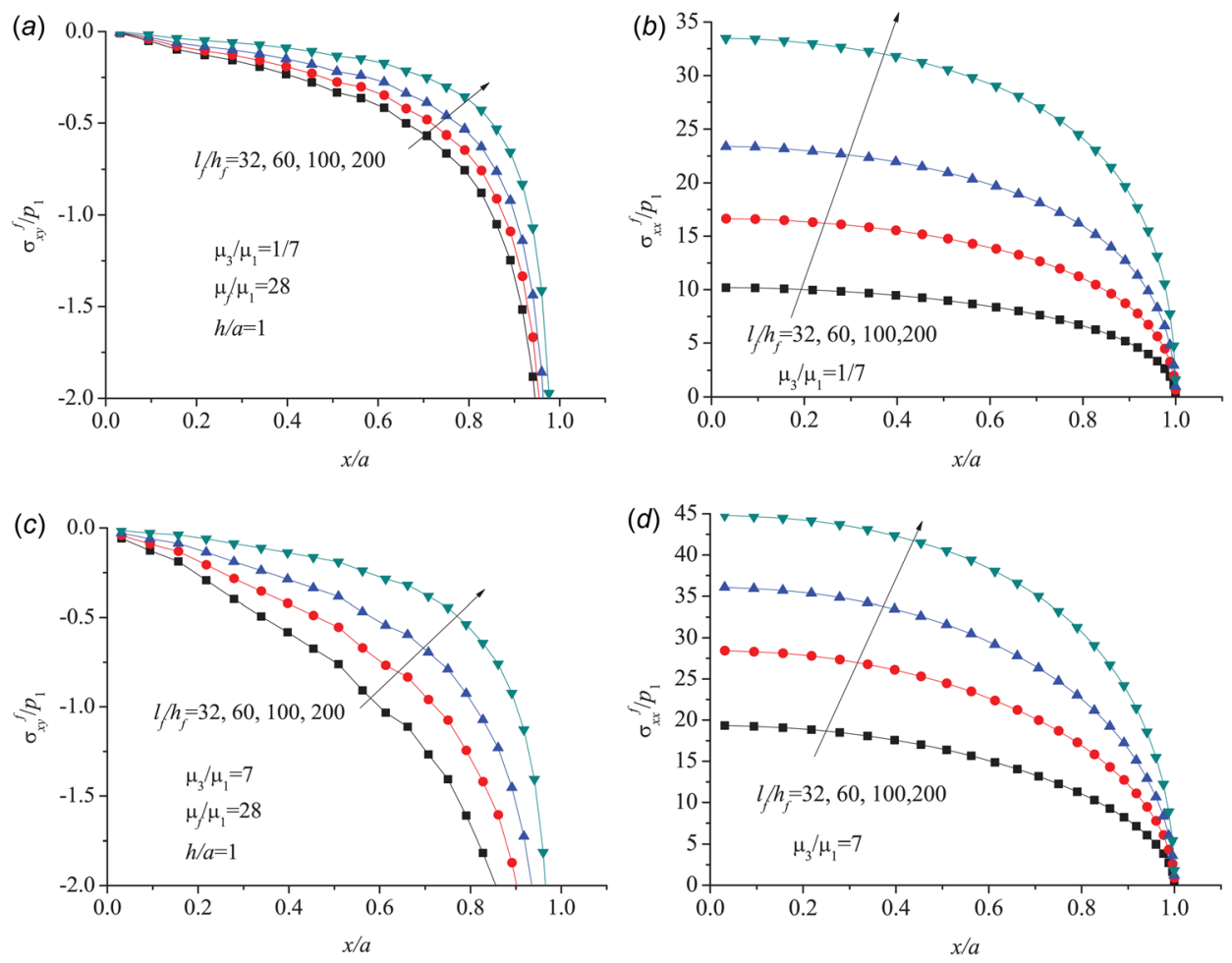

Fig. 8 The distribution of the interface shear stress $\sigma_{x y}^{f} / p_{1}$ and the normal stress in the film $\sigma_{x x}^{f} / p_{1}$ for the model of a deformable film in adhesive contact with a finite-thickness graded substrate with determined parameters $\mu_{f} / \mu_{1}=28, h / a=1$, but with different values $I_{f} / h_{f}$. (a) and $(b)$ for $\mu_{3} / \mu_{1}=1 / 7 ;(c)$ and (d) for $\mu_{3} / \mu_{1}=7$.

Table 3 The stress intensity factors $K_{I I} / p_{1} \sqrt{a}$ near the contact edge versus different ratios $\boldsymbol{I}_{\boldsymbol{f}} / \boldsymbol{h}_{\boldsymbol{f}}$ in cases with determined parameters $\mu_{f} / \mu_{1}=28, h / a=1$, and $\mu_{3} / \mu_{1}$

\begin{tabular}{lcccc}
\hline \hline & $l_{f} / h_{f}=32$ & $l_{f} / h_{f}=60$ & $l_{f} / h_{f}=100$ & $l_{f} / h_{f}=200$ \\
\hline $\begin{array}{l}K_{I I} / p_{1} \sqrt{a} \\
\left.\mu_{3} / \mu_{1}=7\right)\end{array}$ & 1.0831 & 0.9166 & 0.7672 & 0.5738 \\
$\begin{array}{l}K_{I I} / p_{1} \sqrt{a} \\
\left(\mu_{3} / \mu_{1}=1 / 7\right)\end{array}$ & 0.7735 & 0.7048 & 0.6304 & 0.5113 \\
\hline \hline
\end{tabular}

parameters of the film and the graded substrate. Appropriate choice of the film stiffness, the stiffness variation law of the graded substrate, as well as the length scales of the film and substrate, could improve resistance of an interface crack initiation or

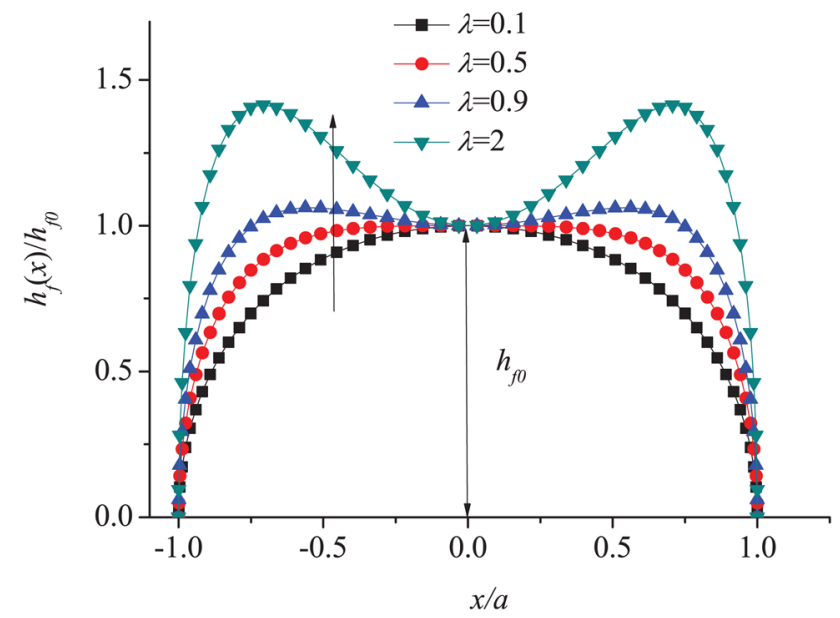

Fig. 9 The profile of the bonded film in the thickness direction with different shape parameters $\lambda$ the film breakage. The results should be helpful for the design of film-substrate systems in practical applications.

\section{Acknowledgment}

P.C. thanks the support of China Postdoctoral Science Foundation Funded Project (No. 2015M580486), NSFC (No. 11402292), Natural Science Foundation of Jiangsu Province (No. BK20140179), Jiangsu Planned Projects for Postdoctoral Research Funds (No. 1501009B), the Fundamental Research Funds for the Central Universities (No. 2014QNA73). S.C. gratefully acknowledges the support by the NSFC (Nos. 11372317 and 11125211), the Nano-project (2012CB937500), and the CAS/SAFEA International Partnership Program for Creative Research Teams.

\section{Appendix}

The kenerls $K_{i j}(x, r)$ in Eqs. (5a) and (5b) are expressed as

$$
\begin{gathered}
K_{11}(x, r)=\int_{0}^{+\infty}\left[\alpha N_{11}(\alpha)+\frac{\kappa+1}{4 \mu_{1}}\right] \sin [\alpha(r-x)] \mathrm{d} \alpha \\
K_{12}(x, r)=-\mathrm{i} \int_{0}^{+\infty}\left[\alpha N_{12}(\alpha)-\mathrm{i} \frac{\kappa-1}{4 \mu_{1}}\right] \cos [\alpha(r-x)] \mathrm{d} \alpha \\
K_{21}(x, r)=-\mathrm{i} \int_{0}^{+\infty}\left[\alpha N_{21}(\alpha)+\mathrm{i} \frac{\kappa-1}{4 \mu_{1}}\right] \cos [\alpha(r-x)] \mathrm{d} \alpha \\
K_{22}(x, r)=\int_{0}^{+\infty}\left[\alpha N_{22}(\alpha)+\frac{\kappa+1}{4 \mu_{1}}\right] \sin [\alpha(r-x)] \mathrm{d} \alpha
\end{gathered}
$$

where $N_{j k}(\alpha)(j, k=1,2)$ are the corresponding four elements in matrix $\mathrm{N}(\alpha)$ 


$$
\begin{aligned}
& \mathrm{N}(\alpha)=\left[\begin{array}{cc}
m_{1} & m_{2} \\
1 & 1
\end{array}\right] \\
& \cdot\left[\begin{array}{cc}
1 /\left(m_{1} n_{1}-\mathrm{i} \alpha\right) & 1 /\left(m_{2} n_{2}-\mathrm{i} \alpha\right) \\
1 /\left\{(\kappa-1)\left[-\mathrm{i} \alpha m_{1}(3-\kappa)+n_{1}(1+\kappa)\right]\right\} & 1 /\left\{(\kappa-1)\left[-\mathrm{i} \alpha m_{2}(3-\kappa)+n_{2}(1+\kappa)\right]\right\}
\end{array}\right]^{-1}
\end{aligned}
$$

Coefficients in Eq. (A2) are as follows:

$$
\begin{aligned}
n_{j}(\alpha)= & -\frac{\gamma}{2}+\sqrt{\frac{\gamma^{2}}{4}+\alpha^{2}-\mathrm{i}(-1)^{j} \gamma \alpha\left(\frac{3-\kappa}{1+\kappa}\right)^{1 / 2}}, \\
& \operatorname{Re}\left(n_{j}\right)>0, \quad j=1,2 \\
n_{j}(\alpha)= & -\frac{\gamma}{2}-\sqrt{\frac{\gamma^{2}}{4}+\alpha^{2}+\mathrm{i}(-1)^{j} \gamma \alpha\left(\frac{3-\kappa}{1+\kappa}\right)^{1 / 2}}, \\
& \operatorname{Re}\left(n_{j}\right)<0, \quad j=3,4
\end{aligned}
$$

and $m_{j}(\alpha)$ for each $n_{j}(\alpha)(j=1, \ldots, 4)$ is

$$
m_{j}(s)=\frac{(\kappa-1)\left(n_{j}^{2}+\gamma n_{j}\right)-\alpha^{2}(\kappa+1)}{\alpha\left[2 n_{j}+\gamma(\kappa-1)\right]}
$$

\section{References}

[1] Freund, L. B., and Suresh, S., 2004, Thin Film Materials: Stress, Defect Forma tion and Surface Evolution, Cambridge University Press, Cambridge, UK.

[2] Chen, H., Feng, X., and Chen, Y., 2014, "Slip Zone Model for Interfacial Failures of Stiff Film/Soft Substrate Composite System in Flexible Electronics,' Mech. Mater., 79, pp. 35-44.

[3] Banerjee, S., and Marchetti, M. C., 2012, "Contractile Stresses in Cohesive Cell Layers on Finite-Thickness Substrates," Phys. Rev. Lett., 109(10), p. 108101.

[4] Akisanya, A. R., and Fleck, N. A., 1994, "The Edge Cracking and Decohesion of Thin Films," Int. J. Solids Struct., 31(23), pp. 3175-3199.

[5] Yu, H. H., He, M. Y., and Hutchinson, J. W., 2001, "Edge Effects in Thin Film Delamination," Acta Mater., 49(1), pp. 93-107.

[6] Alaca, B. E., Saif, M. T. A., and Sehitoglu, H., 2002, "On the Interface Debond at the Edge of a Thin Film on a Thick Substrate," Acta Mater., 50(5), pp. 1197-1209.

[7] Guler, M. A., Gulver, Y. F., and Nart, E., 2012, "Contact Analysis of Thin Films Bonded to Graded Coatings," Int. J. Mech. Sci., 55(1), pp. 50-64.

[8] Arutiunian, N. K., 1968, "Contact Problem for a Half-Plane With Elastic Reinforcement," PMM-J. Appl. Math. Mech., 32(4), pp. 652-665.

[9] Erdogan, F., and Gupta, G. D., 1971, "The Problem of an Elastic Stiffener Bonded to a Half Plane," ASME J. Appl. Mech., 38(4), pp. 937-941.

[10] Erdogan, F., and Civelek, M., 1974, "Contact Problem for an Elastic Reinforcement Bonded to an Elastic Plate," ASME J. Appl. Mech., 41(4), pp. 1014-1018.

[11] Erdogan, F., and Joseph, P. F., 1990, "Mechanical Modeling of Multilayered Films on an Elastic Substrate-Part I: Analysis," ASME J. Electron. Packag., 112(4), pp. 309-316.
[12] Erdogan, F., and Joseph, P. F., 1990, "Mechanical Modeling of Multilayered Films on an Elastic Substrate-Part II: Results and Discussion," ASME J. Electron. Packag., 112(4), pp. 317-326.

[13] Hu, S. M., 1979, "Film-Edge-Induced Stress in Substrates," J. Appl. Phys., 50(7), pp. 4661-4666.

[14] Shield, T. W., and Kim, K. S., 1992, "Beam Theory Models for Thin Film Segments Cohesively Bonded to an Elastic Half Space," Int. J. Solids Struct., 29(9), pp. 1085-1103.

[15] Suresh, S., 2001, "Graded Materials for Resistance to Contact Deformation and Damage," Science, 292(5526), pp. 2447-2451.

[16] Giannakopoulos, A. E., and Suresh, S., 1997, "Indentation of Solids With Gradients in Elastic Properties. 1. Point Force," Int. J. Solids Struct., 34(19), pp. 2357-2392.

[17] Giannakopoulos, A. E., and Suresh, S., 1997, "Indentation of Solids With Gradients in Elastic Properties. 2. Axisymmetric Indentors," Int. J. Solids Struct. 34(19), pp. 2393-2428.

[18] Choi, H. J., and Paulino, G. H., 2008, "Thermoelastic Contact Mechanics for a Flat Punch Sliding Over a Graded Coating/Substrate System With Frictional Heat Generation," J. Mech. Phys. Solids, 56(4), pp. 1673-1692.

[19] Guler, M. A., and Erdogan, F., 2004, "Contact Mechanics of Graded Coatings," Int. J. Solids Struct., 41(14), pp. 3865-3889.

[20] Guler, M. A., and Erdogan, F., 2007, "The Frictional Sliding Contact Problems of Rigid Parabolic and Cylindrical Stamps on Graded Coatings," Int. J. Mech. Sci., 49(2), pp. 161-182.

[21] Ke, L. L., and Wang, Y. S., 2006, "Two-Dimensional Contact Mechanics of Functionally Graded Materials With Arbitrary Spatial Variations of Material Properties,” Int. J. Solids Struct., 43(18-19), pp. 5779-5798.

[22] Ke, L. L., and Wang, Y. S., 2007, "Two-Dimensional Sliding Frictional Contact of Functionally Graded Materials," Eur. J. Mech. A-Solids, 26(1), pp. 171-188.

[23] Mao, J. J., Ke, L. L., and Wang, Y. S., 2014, "Thermoelastic Contact Instability of a Functionally Graded Layer and a Homogeneous Half-Plane," Int. J. Solids Struct., 51(23-24), pp. 3962-3972.

[24] Chen, S. H., Yan, C., and Soh, A., 2009, "Adhesive Behavior of TwoDimensional Power-Law Graded Materials," Int. J. Solids Struct., 46(18-19), pp. 3398-3404.

[25] Chen, S. H., Yan, C., Zhang, P., and Gao, H. J., 2009, "Mechanics of Adhesive Contact on a Power-Law Graded Elastic Half-Space," J. Mech. Phys. Solids, 57(9), pp. 1437-1448.

[26] Guo, X., Jin, F., and Gao, H. J., 2011, "Mechanics of Non-Slipping Adhesive Contact on a Power-Law Graded Elastic Half-Space," Int. J. Solids Struct. 48(18), pp. 2565-2575.

[27] Jin, F., Guo, X., and Gao, H., 2013, "Adhesive Contact on Power-Law Graded Elastic Solids: The JKR-DMT Transition Using a Double-Hertz Model," J. Mech. Phys. Solids, 61(12), pp. 2473-2492.

[28] Guler, M. A., 2008, "Mechanical Modeling of Thin Films and Cover Plates Bonded to Graded Substrates," ASME J. Appl. Mech., 75(5), p. 051105.

[29] Chen, P., and Chen, S., 2013, "Thermo-Mechanical Contact Behavior of a Finite Graded Layer Under a Sliding Punch With Heat Generation,” Int. J. Solids Struct., 50(7), pp. 1108-1119.

[30] Chen, P., Chen, S., and Peng, J., 2015, "Sliding Contact Between a Cylindrical Punch and a Graded Half-Plane With an Arbitrary Gradient Direction," ASME J. Appl. Mech., 82(4), p. 041008.

[31] Gladwell, G. M. L., 1980, Contact Problems in the Classical Theory of Elasticity, Martinus Nijhoff Publishers, The Hague, The Netherlands.

[32] Erdogan, F., Gupta, G. D., and Cook, T. S., 1973, "Numerical Solution of Singular Integral Equations," Methods of Analysis and Solutions of Crack Problems, G. Sih, ed., Springer, Dordrecht, pp. 368-425. 